\title{
8 cosIs: A CONTENT-ORIENTED SHOEPRINT IDENTIFICATION SYSTEM
}

\author{
Mohamed Tahar Meharga, Corinne Plazanet, \\ and Stefano Spaccapietra
}

\author{
Database Laboratory \\ Computer Science Department \\ Ecole Polytechnique Fédérale de Lausanne (EPFL) \\ CH-1015 Lausanne, Switzerland* \\ \{mohamed.meharga, corinne.plazanet, stefano.spaccapietra\}-epfl.ch
}

\begin{abstract}
COSIS is a content-based image retrieval system designed for the shoeprint identification. It consists of a matching-based query tool, an attribute-based query tool and an icon-based browser. The matching-based tool considers the complete and partial shoeprints as query images. The completeshoeprint query tool is fully-automatic whereas the partial-shoeprint one is user-driven. The development of the matching-based query tool is inspired from the template-matching approach largely used in pattern recognition. This paper presents COSIS by focusing, in particular, on its main component: the Matching-based Query Tool.
\end{abstract}

\subsection{INTRODUCTION}

Several products providing the content-based image retrieval (CBIR) functionalities are already available in the marketplace, but since they lack accuracy and the query result is not completely guaranteed, one can never have complete confidence in their conclusions, notably in strategic domains such as identification for access control, forensic science, or medical applications. The main problem is to improve both their efficiency and effectiveness. Further solutions

*This project is partially funded by the Swiss National Science Foundation. 
and advances in indexing and matching efficiency are needed to handle large image databases.

The problem of the similarity perception is complex and depends on the context, the interest and the observer. Query by example is only possible if a constrained view of what is meant by "similar" is defined. Tracking efficiency, our research work has been set up in a concrete application framework, which, at least in a first step, would provide a defined environment as well as a chance to work in close cooperation with users having application expertise and capable of giving us a precise definition of their requirements and direct feedback on our suggestions and results. Thanks to an interesting opportunity, this application domain is the one of criminal investigation, for which the local police maintains a database of images of shoeprints. These black and white binary images also represent a good starting point for such a project: they have reduced, although not minimal, complexity, and allow to focus on shape and texture issues, rather than the more conventional color issues. Moreover, the fact that the number of images is not that large, the relevance of search criteria, similarity measurements and appropriateness of results is easier to assess.

From the user point of view, in our application, each image contains one semantic object: a shoeprint, which can be complete or partial. A semantic object here is defined as an object of the real world, identified by its name in an univocal way.

In object recognition and image understanding, the template matching approach is widely used. To detect the presence of an object in an image, a template of the object is convolved with each pixel of the image and the region that responds the best could correspond to the searched object. The conclusions of the application specification analysis suggested us to investigate this approach and this gave rise to the COSIS system.

In this paper, the Content-Oriented Shoeprint Identification System (COSIS) is described, in particular, its approach to perform the content-based querying. COSIS has three main components. Section 8.2 presents the shoeprint application, the data as well as the retrieval needs and requirements. Section 8.3 exposes a survey of relevant efforts in content based retrieval in general and in forensic science in particular. Section 8.4 discusses the proposed approach for the content-based querying of shoeprint images. Section 8.5 presents the COSIS components. Finally, the Section 8.6 reveals the possible extensions of COSIS to cope with other applications and our future work.

\subsection{APPLICATION AND PROBLEM STATEMENT}

\subsubsection{The data}

One form of physical evidence in judicial investigations is the shoeprint identification. The shoeprints are often encountered at the scene of burglaries and other kinds of crimes. The shoeprints are important for different reasons. They can be found at most of the crime scenes, they are the fastest way to solve a crime, and they are the best way to link crime scenes. 
In a typical situation of police investigations by shoeprints, three main collections of shoeprints are considered: (1) those from shoes of commerce, (2) those from shoes of suspects and (3) impressions gathered from the crime scenes.The main purpose is, using shoeprints from the third category, to look for possible suspects, by getting models and brand names of shoes and to link crime scenes.

For our experiments, our database is composed of more than 800 shoeprints of the category (1). The second category is considered equivalent to the first category. However, the images of the third category are obtained from scanned and binarised grey-level photographs of real impressions.

\subsubsection{Retrieval needs and requirements}

Basically, we have to answer the following query: giving a shoeprint as input, the system has to retrieve similar ones contained in the database. This has to be done with the following considerations:

- Every image (shoeprint) of the database similar to the query one has to be returned by the system. More details are given in the section 8.4 about the meaning of the similarity in this application.

- Invariance is required under translation, scaling, rotation and mirroring.

- The retrieval approach has to be robust to overcome the noise and the artifacts affecting the input images because of the wear of the shoes, the crime scene state, and the conditions under which the impressions are captured. In the gathered impressions, some regions can be absent, dilated, eroded or collapsed with other regions. Moreover, the partial shoeprints have to be taken into account. In several situations, only parts of shoe impressions are gathered from the crime scenes.

\subsection{RELATED WORK}

In the plethora of the proposed CBIR systems (research prototypes or products), we find domain-dependent systems and domain-independent ones. If the first systems lack flexibility and openness, they are more effective and more efficient than the latter ones, since they take advantage of a context (metaknowledge) which is very helpful for the identification of the similarity criteria.

As examples of the first category, we quote ARTISAN [4] and TODAI [5]. ARTISAN is a prototype shape retrieval system for trade mark images (logos). ARTISAN is intended to be a powerful tool for the UK Patent Office Trade Marks Registry. TODAI (Typographic Ornament DAtabases and Identification) is a CBIR system based on the orientation radiograms to describe the content of images. TODAI is used by researchers, working in the area of old books, to consult an International Bank of Printers' Ornaments on the Web.

In the second category, QBIC [6, 7], Virage [8] and Excalibur occupy the head of the list. Virage is perhaps the most interesting. Unlike its rivals, it is designed as a series of independent modules, which can be incorporated within existing database management products to extend their image-handling capabilities to 
content-based retrieval, e.g. VIR datablade in Illustra. In [9], a CBIR system for ophthalmological images is presented. Based on the Virage Incorporated framework, Gupta and al. have designed a number of primitives extracted from ocular fundus images in order to define their similarity for automatic indexing.

Concerning the forensic science community, most (if not all) of the proposed methods (systems) are classification based $[10,13]$. With respect to a specific rules, for each shoeprint in the database is linked a set of geometric shapes (alphanumeric data) as metadata. These metadata are manually introduced by a restrictive group of users who are more experienced and well trained on the system. The classification process requires a coding system which associates for each geometric shape a specific code. The classification codes are divided into main groups and these groups into sub-features. In [12], the major groups are Bars, Circles, Design, Mesh, Pattern, Studs, Waves and Zig-Zags. Each shoeprint is partitioned into four distinct areas of Toe, Ball, Instep and Hill. A set of metadata is associated for each area. Using a graphical interface for questioning the system, the user has to describe the input shoeprint (introduce the metadata). After codification of the geometric shapes proposed by the user and by mean of index the system returns the result ( $a$ list of shoeprints the most similar to the proposed one).

Classifying shoeprints is not an easy task because of the incalculable number of various geometric shapes that are constantly being changed. The manual acquisition of features asks for more objectivity from the group of users responsible of the databases updates which is not an easy task too. It has been also difficult to design a multi-user classification system and a system which perform identification of partial shoeprints.

Among the pertinent research results, conducted by forensic science researchers, obtained up-to-date, the most important one is REBEZO [14]: the first system (in this application) which after segmenting the picture, analyses the geometric shapes (two-dimensional shape analysis with FFT) but the codification is still user-assisted, i.e. the system make only suggestions of the shapes which it recognizes.

\subsection{PROPOSED APPROACH}

\subsubsection{Approach motivations}

Digital image analysis techniques use features (metadata) to classify an image or to segment it. Experience shows that designing appropriate features for a pattern recognition application is the most critical effort to be invested in the course of design. We estimate that, in this project, this step is at least as crucial as in the classical pattern recognition problem, which is related to the issues we want to investigate: binary image retrieval.

One of the key problems in content-based image retrieval is the resolution of the correspondence or similarity between images (or regions) in the both images (query image and database image). There are two broad classes of techniques for similarity measurement: matching-based (area-based) and feature- 
based (indexing-based). In matching-based approach, the intensity of a region around each pixel is taken as the feature and is used so that the regions in both images match. Thus, a local similarity for a region can be found based on measures such as sum of absolute difference of pixel intensity. In the indexingbased approach, features such as the average amount of red, green, and blue in color images ( 3 features per image: $R_{a v g}, G_{a v g}$ and $B_{a v g}$ ), instead of intensity arrays, derived from two images are tested for a similarity.

There are some advantages of indexing-based systems. They are usually faster than matching-based methods since only the features of the target image are calculated at the querying time. The features of the database images are derived at insertion time and stored in indexes which avoids the systematic sweeping of all the database images. They are also less sensitive to photometric variations since they represent (in general) global geometric properties of a scene.

In some cases (particularly when images contain only one semantic feature), the matching-based techniques are more efficient than indexing-based ones since they work directly on the images. However, as they require a sequential scanning of the image database, the system performance is considerably reduced.

After the analysis of an exhaustive sample of the images (the shoeprints , more than 800), we noticed that it is difficult to consider a shoeprint (for about $38 \%$ of the shoeprints) as collection of objects (geometric shapes) with some properties and a specific spatial relationships (Figure 8.1). Furthermore, the use of global features which capture global properties of the picture, as the maxima of orientation radiograms [5], are not appropriate because they are designed for grey-level images. It is better to use information spatially distributed over the object shape rather than at localized portions of the shape. Another aspect
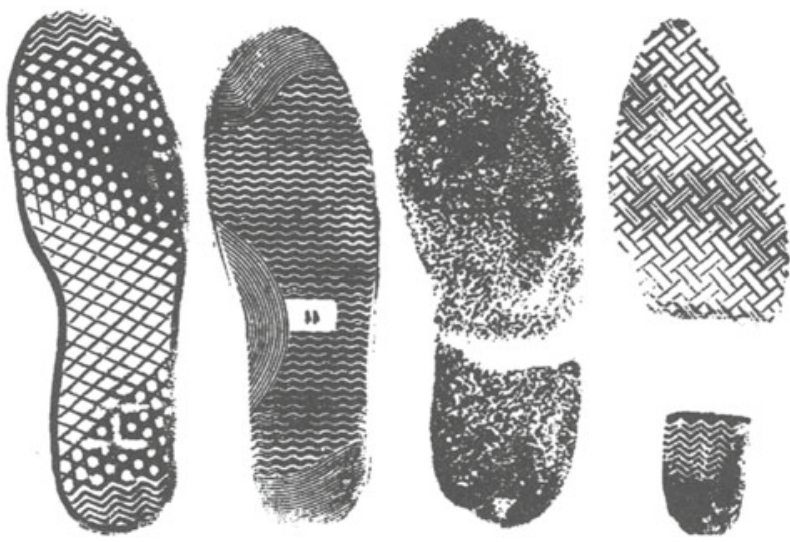

Figure 8.1: Examples of shoeprints.

motivating the proposed approach is that the goal of the retrieval process here is the identification: the images of interest are those corresponding exactly to the 
query one with tolerance of some degree of noise and artifacts. In the example of the Figure 8.2a, the user is interested by the image of the Figure 8.2b and not by the image of the Figure $8.2 \mathrm{c}$ even it is also similar to the query image.
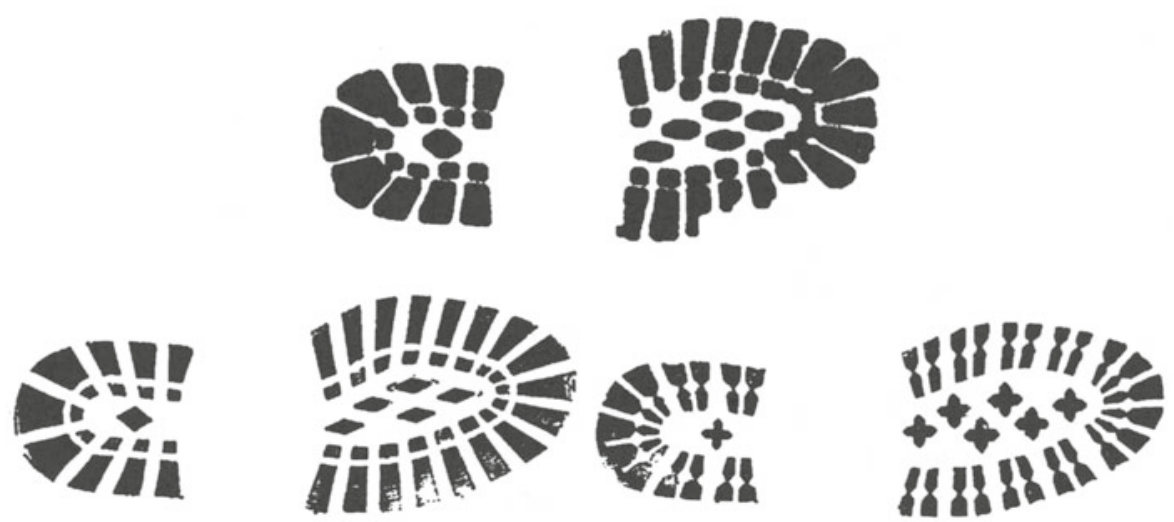

Figure 8.2: Retrieval requirements.

We can distinguish the two kinds of CBIR:

- Similarity oriented retrieval: Among the images of the database, we search images presenting some properties similar to the query image: $I_{d b} \cap I_{q} \neq \emptyset$.

- Identification oriented retrieval: The images of interest are those containing or "equal" to the query image: $I_{q} \subseteq I_{d b} \pm \Delta I$.

Our application falls mainly in the second category.

\subsubsection{The approach}

All the specifications listed above have conducted us to investigate a matchingbased approach. The main idea is to match the two images to compare, by a logical operation, and analyse the difference, i.e. after normalizing the query image (same scale, same position and same direction as the database image), apply a logical difference between the two images and analyse the resulting image. As a simple decision criteria, we can say that the database image the more similar to the query one is the one which has the less number of pixels in the resulting image.

The querying algorithm involves three main steps: 1) preprocessing of the query image, 2) logical operation between the query image and the database images, and 3) analysis of the resulting images. 


\section{Algorithm:}

1st Step: Preprocessing of the query image $\left(I_{q}\right)$

- Shoeprint localization in the image and cropping.

- Standard orientation: the slope of the line of the least squares $(y=$ $A+B * x)$ is used to bring back the shoeprint to a standard orientation,

$$
\begin{gathered}
B=\frac{n \sum x_{i} y_{i}-\left(\sum x_{i} \sum y_{i}\right)}{n \sum x_{i}^{2}-\left(\sum x_{i}\right)^{2}} \\
A=\frac{\sum y_{i}-\left(B \sum x_{i}\right)}{n}
\end{gathered}
$$

$n$ : number of black pixels, $\left(x_{i}, y_{i}\right)$ : coordinates of the $i^{\text {th }}$ black pixel.

- Standard dimensions: As explained above, as the goal of the querying is to retrieve the images that match exactly the query one, this operation doesn't damage the retrieval process since the database images and the query one undergo the same effect of this operation (the same distortion). The database images $\left(I D B=\left\{I_{d b}\right\}\right)$ are preprocessed at insertion time (off-line).

\section{2nd Step: Logical operation}

- An exclusive OR operation is applied between the query image and each image of the database $\left(I_{d}=I_{d b} \oplus I_{q}\right)$ to obtain a set of difference images $\left(I D=\left\{I_{d}\right\}\right)$.

3rd Step: Analysis of the difference images

- The difference images are analyzed to be ranked by their descending similarity degree to the query image. One simple similarity criteria can be the number of pixels in the difference images. The $I_{d}$ that has the less number of pixels could correspond to the $I_{d b}$ which is the most similar to $I_{q}$ and so on. A more sophisticated criteria is the analysis of the connected components (eight connectivity) of the difference images. For each $I_{d}$, the two following parameters are considered:

- NBCC: the number of the connected components that have the size greater than defined threshold,

- SLCC: the size of the largest connected component.

The $I_{d b}$ the most similar to $I_{q}$ corresponds to the $I_{d}$ which has the smallest $(N B C C, S L C C)$. 


\subsection{COSIS COMPONENTS}

COSIS aims at providing various ways to access and retrieve the database images by offering the three following functionalities:

- an Icon-based Browser,

- an Attribute-based Query Tool,

- and a Matching-based Query Tool.

COSIS is accessible from any platform via its Web User Interface. Its interface can be any WWW navigator with JAVA facilities.

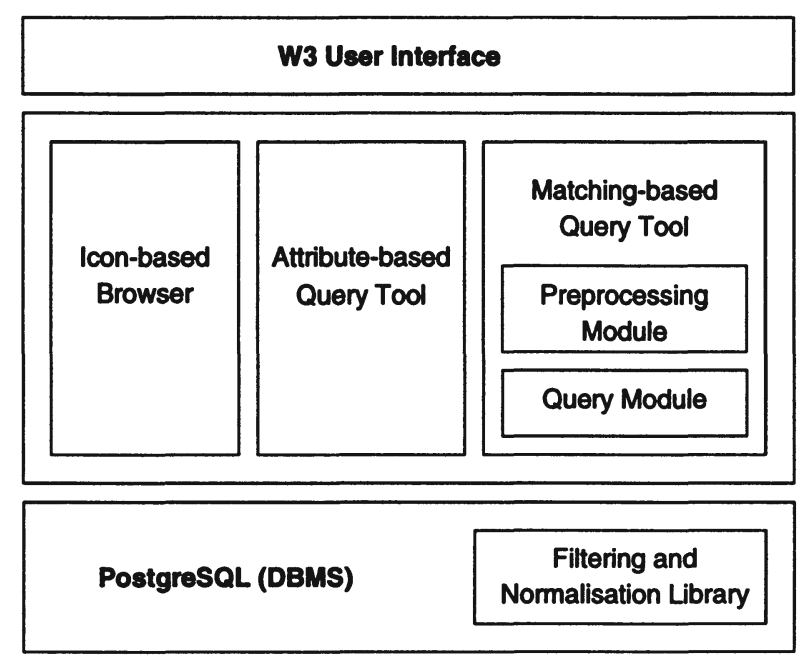

Figure 8.3: COSIS Architecture.

\subsubsection{The Icon-based Browser}

The originality of the Icon-based Browser is that the user, throw his Web Interface, can configure the display of the icon array (the size and the number of icons horizontally and vertically he wants to see at one time). This is performed without causing any distortion to the images. The images are correctly resized to hold within the icon box defined by the user. By clicking on an icon, the corresponding image is displayed in a new window with the list of its attributes (Figure 8.4). Three types of attributes are possible: INTEGER, TEXT and SLIDER. The last attribute was introduced to express the properties that are difficult to specify manually by a number or a string (subjective properties) such as the image luminosity described by an operator. A SLIDER is materialized by a value ( 1 to 100) contained between two bounds (ex. min and max, low and high, etc.). The names of the two bounds are given by the Database Administrator at the creation of the image database. 


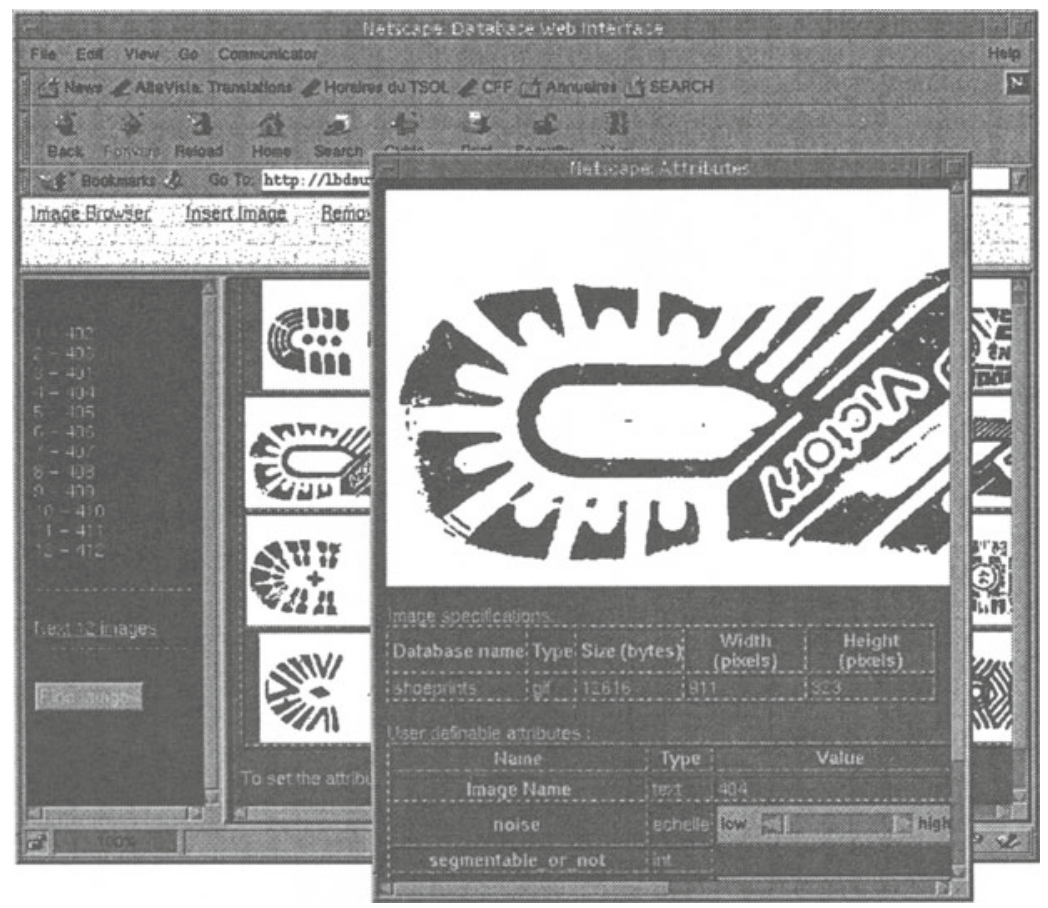

Figure 8.4: The Icon-based Browser.

\subsubsection{The Attribute-based Query Tool}

In the Attribute-based Query Tool, the user is invited to introduce the attributes of the images he is searching. Among the attributes describing the images, for each attribute he is interested by, the user specifies the corresponding value as shown on the Figure 8.5. The result is returned in the Icon-based Browser. As the result consists of a list of images verifying the properties indicated by the user, it would be better viewed by this tool.

\subsubsection{The Matching-based Query Tool}

The Matching-based Query Tool implements the proposed approach presented in section 8.4. The interface uses the file dialog box of the navigator to read the name of the file containing the query image. After the upload of the image, the retrieval process is performed following the algorithm described in the section 8.4 and the result is, as in the Attribute-based Query Tool, returned in the Icon-based Browser. In the example of the Figure 8.6, the query image corresponds to the first image (high-left corner in Figure 8.6b) which has undergone artificially some changes: a spread algorithm, a scaling of factor 0.5 and a rotation of 90 degrees. 


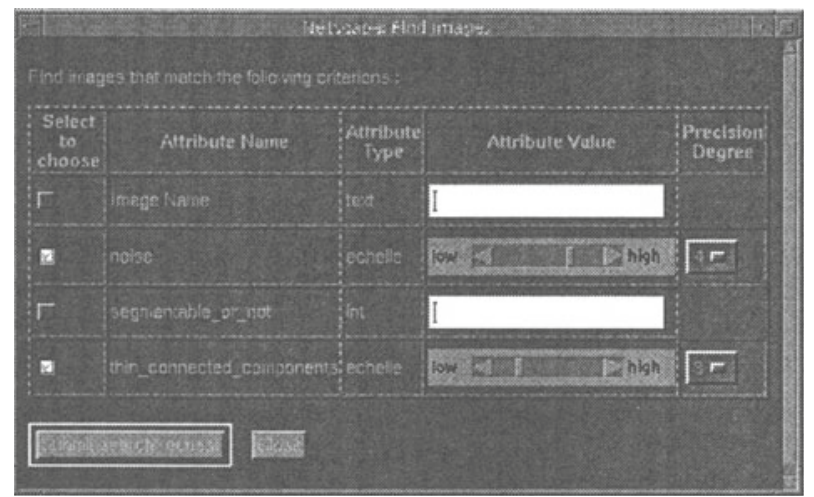

Figure 8.5: Example of attribute-based query.
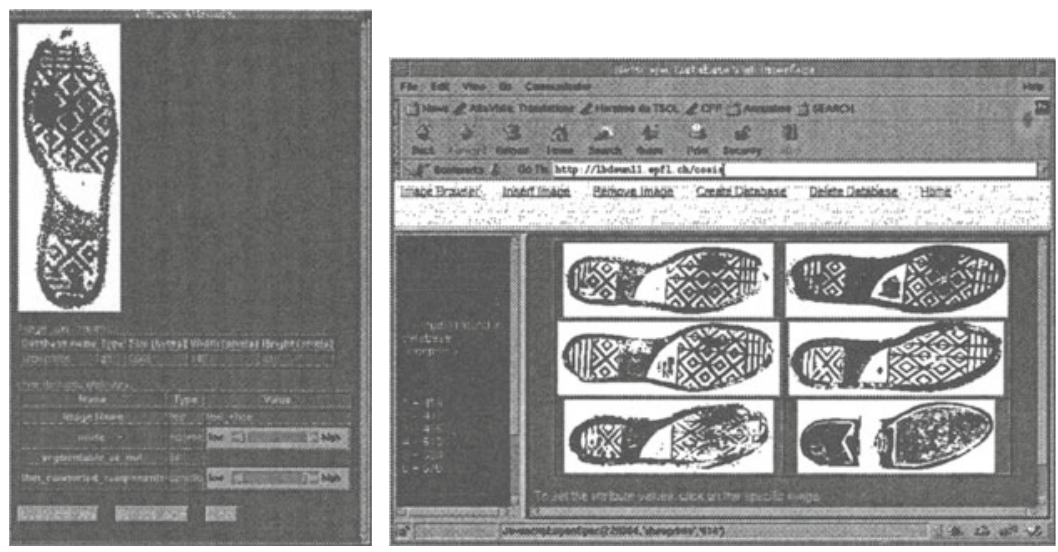

Figure 8.6: Example of matching-based query.

A sample of ten shoeprints is chosen from the database and used as query images in the experiment phase. The images are artificially modified by a graphical editor. They have undergone several changes simultaneously: scaling, rotation and spreading operation (the black uniform regions are split). The Table 8.1 shows the difference between the query image and the returned images for the first images of the result. We clearly see the jump between the first image which is similar to the query one and the rest of the images.

8.5.3.1 Partial shoeprint case. As indicated before, the impressions or the query shoeprints can be only part of shoeprints. The use of the proposed algorithm for these images is obviously not convenient, i.e. we can't convolve a partial object with a complete object as the possible location of the first one 
Table 8.1: Difference between the result images and the query one (average).

\begin{tabular}{lc}
\hline Result image & Difference with the query image $(\%)$ \\
\hline $1^{\text {st }}$ & 12.4 \\
$2^{\text {nd }}$ & 76.1 \\
$3^{\text {rd }}$ & 80.2 \\
$4^{\text {th }}$ & 87.3 \\
$5^{\text {th }}$ & 89.3 \\
$\ldots$ & $\ldots$ \\
\hline
\end{tabular}

in the second one is unknown. For the querying by partial shoeprints we have developed a user-driven algorithm. The idea is to identify first the position of the partial shoeprint in an outline of shoeprint, apply the same algorithm as for the complete shoeprint and using binary mask ignore the rest of the shoeprint.
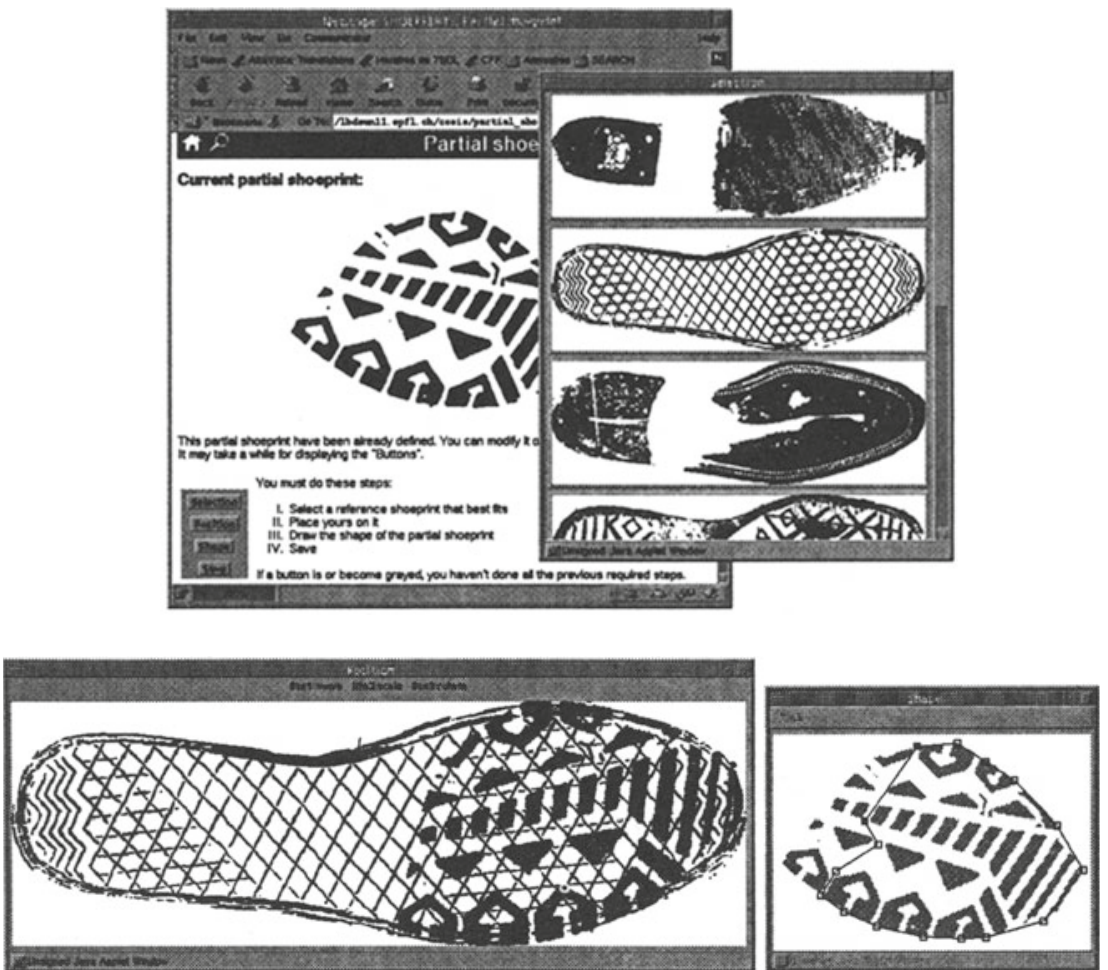

Figure 8.7: Querying with partial shoeprint. 
For the position identification, rather than to scan all the database image (pixel per pixel) to detect which region of the image matches the best the partial shoeprint (as in the template-matching approach in image processing which is very time consuming), this task is delegated to the user. This has been decided after discussion with the concerned users who specified us that, in the majority of the cases, the position of the partial shoeprint in the global outline of a shoeprint is known. This choice is also articulated on the following consideration [6]: "let computers do what they do best - computing - and let humans do what they do best - the positioning in this case which is a quick and non-hard task".

Helped by a user-friendly editor, the user selects first the shoeprint (from a list of representative shoeprints) that the outline may correspond to the searched shoeprint by examining the partial shoeprint (Figure 8.7a). In a second step, he puts the partial shoeprint in the right place of the exampleshoeprint (Figure 8.7b) after what he delimits the region of the partial shoeprint he is interested by (Figure 8.6c) and finally lets the system perform the retrieval.

As the user positioning of the partial shoeprint is not perfect (not trivial task) the results were not as expected. To remedy to this limitation, the input image is convolved ten times with different displacements from the position specified by the user and the position that responds the best is retained. The results were highly improved but still lack accuracy.

\subsection{CONCLUSIONS AND FUTURE WORK}

A matching-based approach to the problem of the shoeprint identification has been proposed. The preliminary results of this approach seems to be very encouraging especially for the complete shoeprints. Normalization is a critical stage of the retrieval process, in particular, invariance to rotation. But the experiments led to very satisfactory results. The benefit to using this approach is that it is still robust for the case where some objects of the query shoeprint are fragmented.

The method may be extended to other applications (binary images) having one semantic object (such as the leaf identification) or considering only one spatial disposition of the image objects. It is developed in our prototype system COSIS. COSIS is implemented on top of the object-relational DBMS PostgreSQL, and its interface can be any WWW browser with JAVA facilities. Users of COSIS may access and retrieve shoeprint images in three different ways.

The matching-based query tool takes for about $2 \mathrm{mn} 20$ " per retrieval in a database of 200 shoeprints. This has to be improved and the future work will include the design of an indexing-based retrieval method to obtain an efficient two-step approach taking advantage of the two concepts: indexing-based and matching-based: 1) coarse retrieval by an indexing-based method to discard the amount of non-candidate images and 2) refinement of the resulting set of images by a matching-based method to maintain only the effective similar images. 


\section{References}

[1] S. Ravela and R. Manmatha. Retrieving Images by Similarity of Visual Appearance. In the Proc. of the IEEE Workshop on Content Based Access of Image Databases, Puerto Rico, June 20, 1997

[2] Stan Sclaroff, Leonid Taycher and Marco La Cascia. ImageRover: A contentbased image retrieval browser for the world wide web. Proc. IEEE Workshop on Content-based Access of Image and Video Libraries, 6/1997

[3] Brian Scassellati, Sophoclis Alexopoulos and Myron Flickner. Retrieving images by 2D shape: a comparison of computation methods with human perceptual judgements. Proceedings of the International Society for Optical Engineering (SPIE), "Storage and Retrieval for Image and Video Databases II". February, 1994

[4] Eakins J P, Boardman J M and Shields K. Retrieval of trade mark images by shape feature - the ARTISAN project. presented at IEE Colloquium on Intelligent Image Databases, London, May 1996

[5] S. Michel, B. Karoubi, J. Bign and S. Corsini. Orientation radiograms for indexing and identification in image databases. in European Conference on Signal Processing (Eupsico), Trieste, 10-13 septembre 1996, pp. 1693-1696

[6] Wayne Niblack, R. Barber, W. Equitz, M. Flickner, E. Glasman, D. Petkovic, and P. Yanker. The QBIC project: Querying images by content using color, texturē, and shape. In Proc. SPIE Electronic Imaging: Science 8 Technology, San Jose, CA February 1993. SPIE

[7] Christos Faloutsos, Ron Barber, Myron Flickner, Jim Hafner, Wayne Niblack, Dragutin Petkovic and Will Equitz. Efficient and Effective Querying by Image Content. Journal of Intelligent Information Systems, 3, 3/4, pp. 231-262, July 1994

[8] Jeffrey R. Bach, Charles Fuller, Amarnath Gupta, Arun Hampapur, Bradley Horowitz, Rich Humphrey, Ramesh Jain, and Chiao-fe Shu. The Virage Image Search Engine: An open framework for image management. Virage, Inc., 1996

[9] Amarnath Gupta, Saied Moezzi, Adam Taylor, Shankar Chatterjee, Ramesh Jain, Michael H. Goldbaum, and S. Burgess. Content-based retrieval of ophthalmological images. In IEEE International Conference on Image Processing, November 1996

[10] Sirkka Mikkonen, Vesa Suominen, and Pia Heinonen. Use of footwear impressions in crime scene investigations assisted by computerized footwear collection system. National Bureau of Investigation, Crime Lab., Finland. Elsevier Science Ireland Ltd, Vol. 82, Issue 1, Sept. 1996

[11] Ch. Belser, M. Ineichen, and P. Pfefferli. Evaluation of the ISAS system after two years of practical experience in forensic police work. Zurich Cantonal Police, Forensic Science Division, Switzerland. Elsevier Science Ireland Ltd, Vol. 82, Issue 1, Sept. 1996 
[12] Wayne Ashley. What shoe was that? The use of computerized image database to assist in identification. Crime Scene Section, Victoria Forensic Science Centre, Forensic Drive, Australia. Elsevier Science Ireland Ltd, Vol. 82, Issue 1, Sept. 1996

[13] Alexandre Girod. Computerized classification of the shoeprints of burglars soles. Police Cantonale Neuchteloise, Service d'Identification Judiciaire, Switzerland. Elsevier Science Ireland Ltd, Vol. 82, Issue 1, Sept. 1996

[14] Zeno Geradts and Jan Keijzer. The image database REBEZO for shoeprints with developments on automatic classification of shoes outsole designs. National Forensic Science Laboratory of the Ministry of justice in the Netherlands, The Netherlands. Elsevier Science Ireland Ltd, Vol. 82, Issue 1, Sept. 1996. 